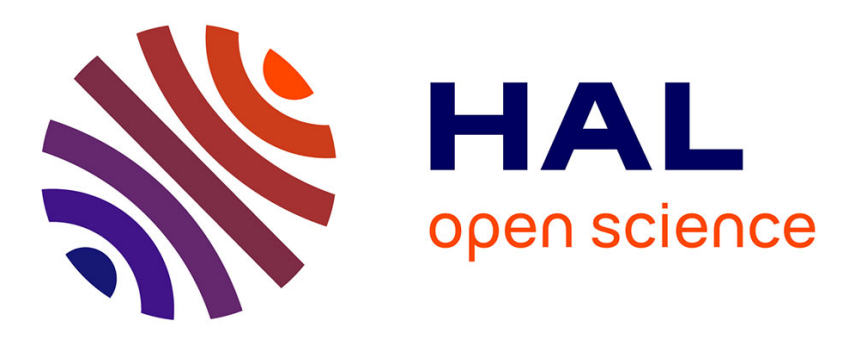

\title{
Bubbles breaking the wall: Two-dimensional stress and stability analysis
}

Jon Alm Eriksen, Benjy Marks, Bjørnar Sandnes, Renaud Toussaint

\section{To cite this version:}

Jon Alm Eriksen, Benjy Marks, Bjørnar Sandnes, Renaud Toussaint. Bubbles breaking the wall: Twodimensional stress and stability analysis. Physical Review E : Statistical, Nonlinear, and Soft Matter Physics, 2015, 91, pp.052204. 10.1103/PhysRevE.91.052204 . hal-01198491

\section{HAL Id: hal-01198491 https://hal.science/hal-01198491}

Submitted on 13 Sep 2015

HAL is a multi-disciplinary open access archive for the deposit and dissemination of scientific research documents, whether they are published or not. The documents may come from teaching and research institutions in France or abroad, or from public or private research centers.
L'archive ouverte pluridisciplinaire HAL, est destinée au dépôt et à la diffusion de documents scientifiques de niveau recherche, publiés ou non, émanant des établissements d'enseignement et de recherche français ou étrangers, des laboratoires publics ou privés. 


\title{
Co \\ Bubbles breaking the wall: Two-dimensional stress and stability analysis
}

\author{
Jon Alm Eriksen* \\ Department of Physics, University of Oslo, P. O. Box 1048 Blindern, N-0316 Oslo, Norway \\ and Institut de Physique du Globe de Strasbourg, University of Strasbourg/EOST, CNRS, 5 rue Descartes, F-67084 Strasbourg Cedex, France \\ Benjy Marks \\ Department of Physics, University of Oslo, P. O. Box 1048 Blindern, N-0316 Oslo, Norway \\ and College of Engineering, Swansea University, Singleton Park, SA2 8PP Swansea, United Kingdom \\ Bjørnar Sandnes \\ College of Engineering, Swansea University, Singleton Park, SA2 8PP Swansea, United Kingdom \\ Renaud Toussaint \\ Institut de Physique du Globe de Strasbourg, University of Strasbourg/EOST, CNRS, 5 rue Descartes, F-67084 Strasbourg Cedex, France \\ and Department of Physics, University of Oslo, P. O. Box 1048 Blindern, N-0316 Oslo, Norway
}

(Received 13 March 2015; published 26 May 2015)

\begin{abstract}
Submerged granular material exhibits a wide range of behavior when the saturating fluid is slowly displaced by a gas phase. In confined systems, the moving interface between the invading gas and the fluid/grain mixture can cause beads to jam, and induce intermittency in the dynamics. Here, we study the stability of layers of saturated jammed beads around stuck air bubbles, and the deformation mechanism leading to air channel formations in these layers. We describe a two-dimensional extension of a previous model of the effective stress in the jammed packing. The effect of the tangential stress component on the yield stress is discussed, in particular how arching effects may impact the yield threshold. We further develop a linear stability analysis, to study undulations which develop under certain experimental conditions at the air-liquid interface. The linear analysis gives estimates for the most unstable wavelengths for the initial growth of the perturbations. The estimates correspond well with peak to peak length measurements of the experimentally observed undulations.
\end{abstract}

DOI: 10.1103/PhysRevE.91.052204

PACS number(s): 45.70.Vn, 47.56.+r, 46.32.+x

\section{INTRODUCTION}

Multiphase flow involving unconsolidated granular media and granular-fluid mixtures occur in a wide range of environmental and engineered processes. Examples include gas venting in sediments, volcanic eruptions, soil wetting and drying, oil and gas recovery, hydraulic fracturing, and carbon geosequestration [1-6]. Similar flow systems are also attracting an increasing scientific interest. A range of flow behaviors have been observed, including destabilized viscous fingers [7], granular decompaction fingers [8-10], channeling [1,11], gas expulsion of imbibated nanoparticle aggregates [12], aerofractures [13,14], and fractures involving immiscible fluids [15-19]. In particular, when a layer of granular material accumulates at the fluid interface, a rich set of flow morphologies have been observed [20], such as labyrinth patterns of frictional fingers [21,22], frictional fingers aligned by gravity [23], and bubble structures [15,24]. Examples of frictional fingers and bubble patterns are shown in Fig. 1.

Consider a horizontal Hele-Shaw cell, filled with a liquid mixture containing beads which sediment out of the liquid. Air is compressed into the cell. The compression rate is so slow $(0.01-0.03 \mathrm{ml} / \mathrm{min})$ that the process can be considered quasistatic. The air displaces the liquid mixture in small intermittent incremental steps. The invading air-liquid inter-

*Corresponding author: jonaerik@fys.uio.no face bulldozes up the beads from the sedimented region, and accumulates the beads into a compacted region adjacent to the air-liquid interface. This accumulated region will in the following be referred to as the front.

An important control parameter for the experiment is the normalized filling fraction, $\phi$, i.e., the height of the sedimented region relative to the cell height. This parameter determines the rate of accumulation of new beads onto the front, as the air-liquid interface advances. As the pattern develops, most of the front is jammed, and only a small section of the interface evolves in intermittent, stick-slip-like increments. For $\phi<0.4$, the system generates treelike structures of frictional fingers [see Fig. 1(a)], with a characteristic finger width [21,22]. When the filling fraction $\phi$ increases, the displaced volume per increment also increases, and the increment frequency decreases. The increments start to form bubbles, rather than small deformations at a finger tip, and the remaining pattern consists of a series of bubbles connected by thin channels of air [24] [see Figs. 1(b) and 1(c)]. After a bubble is formed, the front around the bubble settles down in a static configuration. As the pressure increases beyond a certain level, the front slowly deforms and undulations along the air-liquid interface develop. One of the peaks of these undulations gets ahead of the others and forms a narrow channel through the front. Once the channel approaches the sedimented region, it accelerates, and bursts into a new bubble. A closeup picture of the structure of the bubbles is shown in Fig. 2; videos of the dynamics are provided as Supplemental Material [25]. 


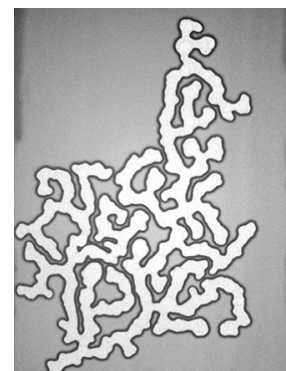

(a)

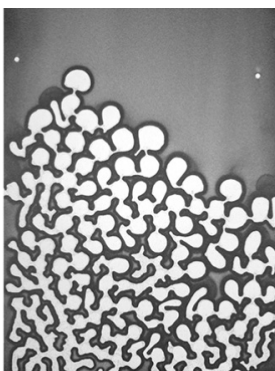

(b)

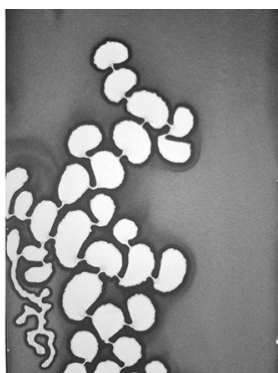

(c)
FIG. 1. The Hele-Shaw cell as seen from above $(20 \times 30 \mathrm{~cm})$. Air (white region) is injected into a liquid mixture with a layer of sedimented beads on the bottom plate (gray region). The front is the accumulated region of beads along the air interface (the dark rim around the white regions). The different images correspond to different normalized filling fractions $\phi$, i.e., the height of the sedimented layer relative to the cell gap. (a) $\phi=0.35$, (b) $\phi=0.49$, and (c) $\phi=0.53$. We see a gradual transition from frictional fingers (a) to bubble dynamics (c) as $\phi$ increases.

The frictional finger behavior gradually transitions into the bubble behavior, either by increasing $\phi$ or the volume of the air in the syringe pump used to compress the air. The transition, and the experimental conditions, are described in detail in Ref. [15].

The dynamics of the finger behavior is understood, at least to the extent that the patterns can be reproduced by simulations. The patterns are simulated both for a horizontal cell [21,22], and for a tilted cell [23], where gravitational effects also are

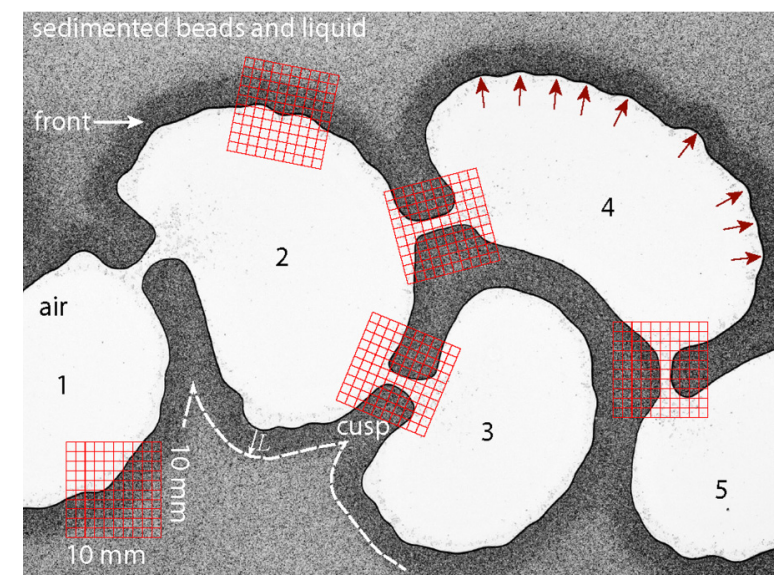

FIG. 2. (Color online) A closeup view of connected bubbles of air, which displace a liquid containing glass beads. The front is the accumulated region of beads adjacent to the air interface, and is identified as the dark region. The white dashed line indicates parts of the separation path between the front and the sedimented region. This path can develop cusps, as front segments from different bubbles merge. The front thickness $(L)$ is only defined where this separation path runs parallel to the air-front interface. Grids of $1 \mathrm{~mm}$ spacing, are superposed on the image to reveal the scales. The front thickness $(L)$ is $\simeq 3 \mathrm{~mm}$ thick. The channels that connect the bubbles are $\simeq 1 \mathrm{~mm}$. The cell gap is $0.5 \mathrm{~mm}$, and the bead diameter is $0.1 \mathrm{~mm}$. The numbers refer to the order in which the bubbles are formed. The air-front interface of the bubble develops undulations. The arrows in bubble 4 points to peaks of these undulations.

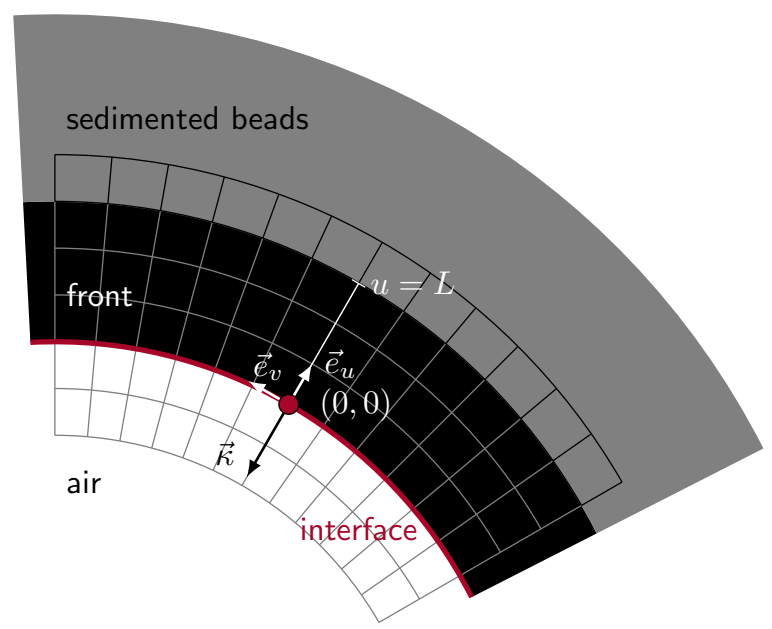

FIG. 3. (Color online) Schematics of a section of the interface, with the adjacent front, seen from above. At every point along the interface we introduce a coordinate system $(u, v)$, such that the point is placed in the origin. The unit vectors $\vec{e}_{u}$ and $\vec{e}_{v}$ point respectively perpendicular and parallel to the interface.

present. Central to the theoretical understanding is a model of the effective stress in the front. In particular, the description of how the stress component normal to the air-liquid interface gives rise to frictional stresses along the plate boundaries. This model accounts for neither the curvature of the front in the expression for the effective stress nor the tangential stress component. It is, however, reasonable to assume that the tangential stress becomes important for highly curved interfaces, which indeed are present in the experimental observations of the bubble behavior, in particular around the channels which connect the bubbles (see Fig. 2).

The aim of this paper is twofold. First, we present a natural extension of the stress model, which also accounts for the curvature of the interface and the tangential stress component inside the packing. We will assume that the tangential and the normal stresses are linearly related. This assumption implies that the tangential stress can have a large impact on the yield stress of the interface. We will also discuss how arching effects are captured by the model, and how they may be important for describing the dynamics of the interface as it moves through the front of a bubble.

Second, we present a linear stability analysis of the deformations at the interface. This analysis gives predictions for the most unstable wavelength of the interface, which agrees well with the experimentally observed peak to peak distance of the undulations.

\section{THEORETICAL CONSIDERATIONS}

There are two local variables along the interface which are of special interest. One is the in-plane signed curvature of the air-liquid interface, $\kappa= \pm|\vec{\kappa}|$, where $\vec{\kappa}$ is the curvature vector, shown in Fig. 3. Note that, while the air-liquid interface may be convoluted at the scale of a single bead, we are here interested in the curvature of the averaged interface, at the scale of several neighboring beads. The absolute value of the curvature is reciprocal to the radius of curvature, $|\vec{\kappa}|=R^{-1}$, 


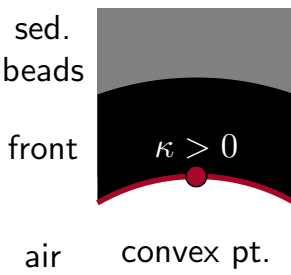

(a)

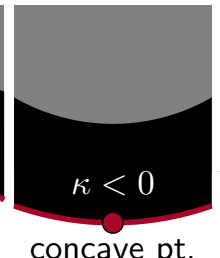

(b)

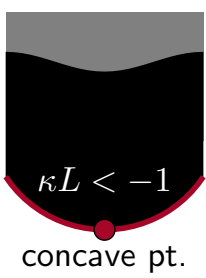

(c)
FIG. 4. (Color online) Schematic examples of different configurations. (a) Convex interface, positive curvature. (b) Concave interface, negative curvature. (c) Negative curvature with radius of curvature which is smaller than the front thickness.

and its sign is defined to be positive if the radius of curvature can be drawn into the air phase, and negative otherwise. Examples of different configurations are shown in Fig. 4. The other variable of interest is the thickness of the front in the direction perpendicular to the interface, $L$, indicated in Figs. 2 and 3. This variable is, however, not applicable to every point along the interface. For example, if two front segments from different sections of the interface merge together, the corresponding sections of the interface stagnate, and remain inactive in the subsequent evolution of the interface (see for example the front enclosed between the bubbles labeled 3 and 4 in Fig. 2). Note also that the separation path between the front and the sedimented region (see the white dashed line in Fig. 2) may develop singular points (cusps), as it evolves, in contrast to the air-front interface which appears smooth everywhere due to the effective surface tension. The front thickness $L$ is only defined where the separation path between the front and the sedimented region runs parallel to the air interface. We will, in the subsequent discussion, only consider points along the interface where $L$ can be defined.

It is convenient to introduce a set of coordinates relative to the points along the interface. Let $(u, v)$ be an orthogonal coordinate system, such that $u$ runs in the direction perpendicular to the interface, and $v$ runs parallel, as shown in Fig. 3. The interface in a small neighborhood around a given point is therefore approximated by $(0, v)$. The separation between the front and the sedimented region, if it exists at that point, is approximated by $(L, v)$. The $(u, v)$ coordinates will be Cartesian around straight segments and inflection points, i.e., points which correspond to $R \rightarrow \pm \infty$, or $\kappa \rightarrow 0$. For straight segments we have that $u=x$, and $v=y$ in the notation used in Ref. [22]. We can identify a polar coordinate system $(r, \theta)$, at curved segments, with origin at the center of the circle corresponding to the radius of curvature, such the interface, is located at $r=R$. The separation path between the front and the sedimented region is located at $r=R+L$ for positively curved segments, and at $r=R-L$ for negatively curved segments. We have the following transformations:

$$
\begin{aligned}
& r=\operatorname{sgn}(\kappa) u+R, \\
& \theta=\frac{\operatorname{sgn}(\kappa) v}{R},
\end{aligned}
$$

where sgn is the sign function. Front lengths which are longer than the radius of curvature, $L>R$, when the interface curves negatively, are not properly accounted for [see Fig. 4(c)]. These

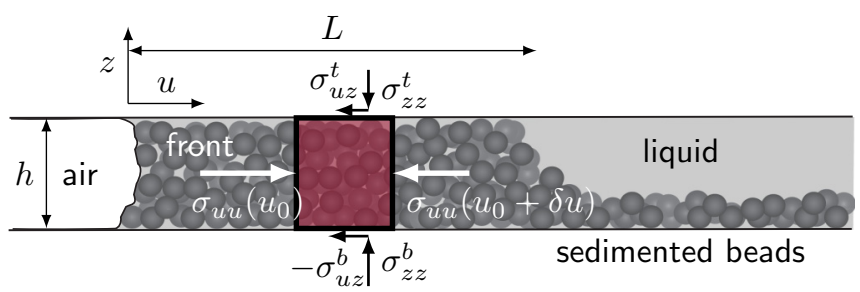

FIG. 5. (Color online) Schematic cross section of the cell at the front. The front thickness $(L)$ is defined to be the length of the region of beads which fills the whole cell gap.

points are rare, and we assume that they are immobile. We will also use $z$ as the coordinate of the height direction, such that the bottom boundary is located at $z=0$, and the top boundary at $z=h$; see Fig. 5 .

\section{A. Yield pressure at the interface without tangential stresses}

We will in the following first review a simplified version of the stress model used in Refs. [21,22]. This derivation will naturally motivate the inclusion of the tangential stress, presented in the next subsection.

The yield pressure associated with a deformation of a section of the interface, i.e., the air pressure at which a section of the front transitions from a sticking to a slipping state, arises from two different effects. First, the air-liquid surface tension of the menisci between the beads will generate an effective surface energy at the scale of several neighboring beads. This surface energy generates a surface stress which acts to minimize the curvature, $\kappa$. The pressure difference which corresponds to the effective surface tension $\gamma$ is given by $\gamma \kappa$.

Second, force chains in the front transmit stresses from the boundary of the cell to the beads at the interface, resulting in an effective normal stress at the interface of the bead packing. Let $\sigma$ be the effective stress tensor field in the bead packing, which we assume to be smooth and continuous at the scale of several bead diameters. We employ a positive sign convention for compressive stresses, and we ignore the $z$ dependence in the stress field, i.e., we consider height averaged stresses. We further assume that variations in the $v$ dependence are negligible, such that $\sigma=\sigma(u)$. The normal stress at the interface is denoted $\sigma_{u u}(u=0)$.

Previous papers $[15,21,22]$ have, in the context of frictional fingers, successfully modeled the yield pressure at the interface by assigning an $L$-dependent yield threshold, $\sigma_{Y}(L)$, to the effective bead stress, such that a local section of the interface evolves if $\sigma_{u u}(u=0)>\sigma_{Y}(L)$. The slip criterion for a section of the interface is

$$
p>\gamma \kappa+\sigma_{Y}(L),
$$

where $p$ is the air pressure. Note that we have ignored the pressure drop associated with the curvature in the out-of-plane direction on the right hand side of the inequality. This pressure drop is, however, constant along the interface, and does not change the location of the weakest section. In order to describe how the threshold $\sigma_{Y}(L)$ depends on the front thickness $L$ we 
first describe how the effective stress field varies through the front.

Consider a straight segment of the front $(\kappa=0)$ such that the $(u, v)$ coordinates are Cartesian. Imagine a representative elementary volume in the front which is bounded by $u_{0}<u<$ $u_{0}+\delta u$ and $0<v<\delta v$. The volume fills the height of the cell such that $0<z<h$. A cross section of this volume is shown by the black square region in Fig. 5. The force balance of the $u$ component of the force on this volume gives us a differential equation for $\sigma_{u u}(u)$,

$$
\begin{aligned}
h \delta & v\left[\sigma_{u u}\left(u_{0}\right)-\sigma_{u u}\left(u_{0}+\delta u\right)\right] \\
& =\delta u \delta v\left[-\sigma_{u z}^{b}\left(u_{0}\right)+\sigma_{u z}^{t}\left(u_{0}\right)\right] \\
& \Rightarrow \frac{\partial}{\partial u} \sigma_{u u}(u)=-\frac{1}{h}\left[-\sigma_{u z}^{b}(u)+\sigma_{u z}^{t}(u)\right]=-F,
\end{aligned}
$$

where $\sigma_{u z}^{b}$ and $\sigma_{u z}^{t}$ are the $u$ components of the shear stresses acting on the bottom and the top cell boundaries respectively, as shown in Fig. 5. In the last equation we also introduce the force density $F$ for later convenience.

To close the system, we need to approximate how these shear stresses change with $u$. We will, as [21,22], follow Janssen's analysis for stresses in a silo [26], which rests on the following two assumptions. First, we assume that the stresses in the $z$ and the $u$ directions are proportional,

$$
\sigma_{z z}^{b}=K_{1} \sigma_{u u} \quad \text { and } \quad \sigma_{z z}^{t}=K_{1} \sigma_{u u},
$$

where $K_{1}$ is the Janssen parameter [27]. We ignore the contribution of the weight of the beads on the bottom plate, which induces an asymmetry in the comparison of the top and bottom boundaries. This contribution was accounted for in the expression developed in Ref. [22], but gives only a minor correction to the exponential $L$ dependence of $\sigma_{Y}(L)$, described below. Second, we assume that the frictional stresses are proportional to the normal stresses acting on the plates (see Fig. 5), i.e., we assume Coulomb friction. The maximum frictional stresses at the plate boundaries are given by $\sigma_{u z}^{t}=\mu \sigma_{z z}^{t}$ and $\sigma_{u z}^{b}=-\mu \sigma_{z z}^{b}$, where $\mu$ is the static friction coefficient. The result of the above assumptions is that

$$
F=\frac{2 \mu K_{1}}{h} \sigma_{u u} .
$$

Limitations of Janssen's assumtions [Eq. (4)] is discussed in $[28,29]$. The result of using a relation like Eq. (5) is, however, in accordance with experimental evidence in the context of frictional fingers [22], for aerofractures [8,13,14] and for the original application of the stresses in silo geometries [26,30].

We also assume that the maximum stress the beads at the end of the front (i.e., at $u=L$ ) can withstand before the front segment slips is a constant $\sigma_{T}$, i.e., the front slides if $\sigma_{u u}(u=$ $L)>\sigma_{T}$. Note that this constant is assumed to be independent of the local parameters, $\kappa$ and $L$, of the interface. Using $\sigma_{T}$ as a boundary condition at $u=L$, we can integrate equation Eq. (3) and get

$$
\sigma_{u u}(u)=\sigma_{T} e^{-2 \mu K_{1}(u-L) / h},
$$

which corresponds to the normal stress profile through the front at the yield transition. It is convenient to introduce a
TABLE I. Approximate values of the parameters of the model.

\begin{tabular}{lccc}
\hline \hline Parameter & & Value & Units \\
\hline Effective surface tension & $\gamma$ & 60 & $\mathrm{mN} / \mathrm{m}^{\mathrm{a}}$ \\
Characteristic length & $\xi$ & 0.06 & $\mathrm{~cm}^{\mathrm{b}}$ \\
Threshold at end of front $(u=L)$ & $\sigma_{T}$ & 10 & $\mathrm{~Pa}^{\mathrm{c}}$ \\
\hline \hline
\end{tabular}

${ }^{a}$ This is lower than the table value of glycerol-water mixture (65-70 $\mathrm{mN} / \mathrm{m}$ ). Note that the complex geometry of the air-liquid interface, due to the menisci between the beads, may change the effective surface tension from that of a pure liquid value. This value has, however, been used to reproduce the finger structures in simulations $[22,23]$.

b $\xi=h /\left(2 \mu K_{1}\right)$. Assuming friction constant for glass beads, $\mu=0.5$ and the Janssen parameter $K_{1}=0.8$ [22]. The cell height is $h=0.05$ $\mathrm{mm}$.

${ }^{\mathrm{c}}$ Order of magnitude estimate based on the average over-pressure presented in [15].

characteristic length,

$$
\xi=\frac{h}{2 \mu K_{1}} .
$$

If we evaluate Eq. (6) at the interface $(u=0)$, we get the final expression for the yield stress,

$$
\sigma_{Y}(L)=\sigma_{T} e^{L / \xi} .
$$

Inserting this into Eq. (2), gives

$$
p>\gamma \kappa+\sigma_{T} e^{L / \xi} .
$$

The weakest section along the interface is identified by having $\kappa$ and $L$ such that $\gamma \kappa+\sigma_{T} \exp (L / \xi)$ is minimal. This criterion is used to simulate fingering behavior in Refs. [21,22], with the exception of the correction term for the weight of the grains mentioned above. $\sigma_{T}$ can be estimated from the friction of the weight of the grains at the transition between the front and the sedimented beads, assuming a wedgelike bead profile [22]. Note also that the numerical value of $K_{1}$ is hard to determine, as it only appears multiplied with the friction coefficient $\mu$ in Eq. (7). Approximate values for the different parameters are listed in Table I. In the context of fingers in a tilted cell [23], it was adequate for the level of detail in the simulationexperiment comparison, to linearize the exponential behavior, i.e., using the first order term from the $L$ expansion of $\sigma_{Y}(L)$ in Eq. (2).

\section{B. Including the tangential stress component}

Equation (8) is a reasonable approximation as long as the curvature is small relative to the front thickness $\kappa L \ll 1$. We therefore do not expect it to hold in the context of bubble formation, as the geometry of the front curves significantly, especially near the channels between the bubbles. A closer inspection will reveal that $\sigma_{Y}$ in Eq. (8) also depends on the curvature, $\sigma_{Y}=\sigma_{Y}(L, \kappa)$.

We can write the mechanical equilibrium in Eq. (3) in a more general form,

$$
\operatorname{div} \sigma=\nabla \cdot \sigma^{T}=-F \vec{e}_{u},
$$


where $F$ is defined in Eq. (3) and $\vec{e}_{u}$ is the unit vector in the $u$ direction. We have that the $r$ component of Eq. (10), in the cylindrical coordinates introduced in Eq. (1) is (see for example Chap. 2 in Ref. [31])

$$
\begin{aligned}
& \frac{1}{r} \partial_{r}\left(r \sigma_{r r}\right)- \frac{\sigma_{\theta \theta}}{r}=-F, \\
& \Rightarrow \partial_{r} \sigma_{r r}=-\frac{\sigma_{u u}}{\xi}-\frac{\sigma_{r r}-\sigma_{\theta \theta}}{r},
\end{aligned}
$$

where we use Eqs. (5) and (7) to substitute for $F$ in the last line. Since we already assume a Janssen approximation for the normal stress in the $z$ direction, it is reasonable to also assume a similar linear relationship for the normal stress in the $\theta$ direction. Note that $r$ and $\theta$ are the principal directions of the stress tensor, due to the symmetry of the annulus (see Fig. 3). Analogous to Eq. (4), we assume that

$$
\sigma_{\theta \theta}=K_{2} \sigma_{r r} .
$$

The principal stresses are thereby assumed to be linearly dependent on each other, but note that the $K_{1}$ parameter is used in a height averaged setting in Eq. (4), whereas $K_{2}$ relates $\sigma_{r r}$ to $\sigma_{\theta \theta}$ everywhere in the $(r, \theta)$ plane. The assumption of a local linear relation between the principal stresses is also used to describe stress distributions in piles of granular material [32-34].

Using the linear dependence assumption in Eq. (13), we can rewrite Eq. (12) as

$$
\partial_{r} \ln \sigma_{u u}(r)=-\frac{1}{\xi}-\frac{1-K_{2}}{r} .
$$

Assume that the interface is positively curved, such that the interface is located at $r=R$, and the end of the front at $r=R+L$. Integrating this, with similar boundary conditions as before, $\sigma_{u u}(R+L)=\sigma_{T}$, and evaluating $\sigma_{r r}(r)$ at $r=R$, gives the yield stress, $\sigma_{Y}(L, \kappa)$. We have that

$$
\sigma_{Y}(L, \kappa)=\sigma_{T} e^{L / \xi}(1+\kappa L)^{1-K_{2}},
$$

where we use $\kappa=1 / R$. One can verify that we obtain the same result if we instead consider a negatively curved section of the interface.

If we use the expression for the effective yield stress which incorporates the radial stress contribution [Eq. (15)] in the previous yield criterion [Eq. (2)], we finally have the new yield criterion,

$$
p>\gamma \kappa+\sigma_{T} e^{L / \xi}(1+\kappa L)^{1-K_{2}} .
$$

Note that if we only consider the correction to Eq. (8) in the radial direction, i.e., we disregard the $\sigma_{\theta \theta}$ contribution by setting $K_{2}=0$ in Eq. (15), the correction will always give a higher yield stress for positive curvature. If we set $K_{2}=1$, i.e., we consider isotropic stress in the $(u, v)$ plane, we get the same stress law as in the one-dimensional model approximation in Eq. (8).

It is hard to estimate the value of $K_{2}$ in our experiments. Its local value may be very sensitive to how the beads are compacted, and may also vary along the interface. In the context of a silo geometry, Janssen coefficient less than 1 and greater than 1 have been reported, depending on the packing procedure [30]. Note that the yield stress changes
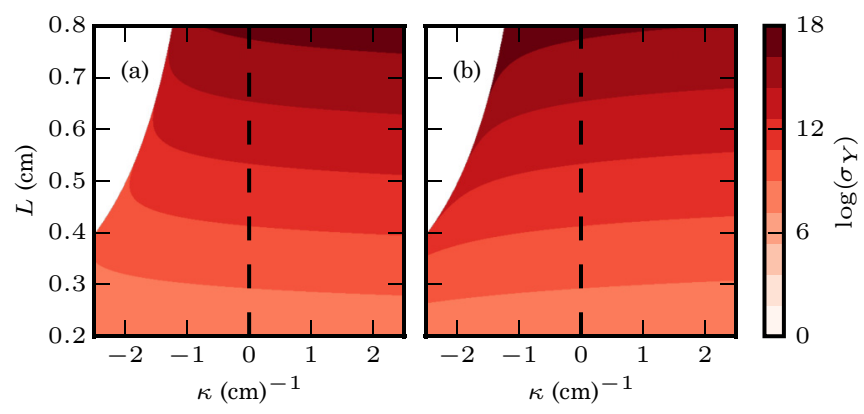

FIG. 6. (Color online) Effective yield stress, $\sigma_{Y}(\kappa, L)$, defined in Eq. (15), for values of $K_{2}$, less than and greater than 1. (a) $K_{2}=$ 0.6 . (b) $K_{2}=1.4$. The contour lines are logarithmically spaced. The threshold increases as Eq. (8), along the dashed line $(\kappa=0)$. The white region in the top left corner corresponds to $\kappa L<-1$, and is not accounted for by the theory. The numerical values of the other parameters are presented in Table I.

qualitatively as $K_{2}$ grows beyond 1, as illustrated in Fig. 6, and that $K_{2}>1$ naturally describes arching mechanisms in the front for negatively curved segments. We will in the following assume that $K_{2}<1$ for straight segments $(\kappa \simeq 0)$; this is in agreement with numerical estimates from discrete element method simulations, $K_{2} \simeq 0.8 \pm 0.1$ [35]. We will further discuss $K_{2}$ in light of the subsequent stability analysis and how it may change with the curvature in Sec. III.

\section{Linear stability analysis of a straight front segment}

We will in the following present a stability analysis by considering perturbations of a straight interface, with a constant front thickness $L$. Consider an infinitesimal perturbation $f_{q}(x)$, with wave number $q$, such that

$$
f_{q}(x)=\epsilon[1+\cos (q x)] .
$$

This perturbation is shown in Fig. 7. The perturbation amplitude $\epsilon$ is infinitesimal. Note that we only consider displacement towards the front, in the positive $y$ direction, $f_{q}(x) \geqslant 0$.

A reasonable condition for growth of a perturbation can be based on the static properties of the front. We assume that the perturbation grows if the threshold at the peaks of the perturbations, i.e., at $\cos (q x)=1$, is lower than the threshold

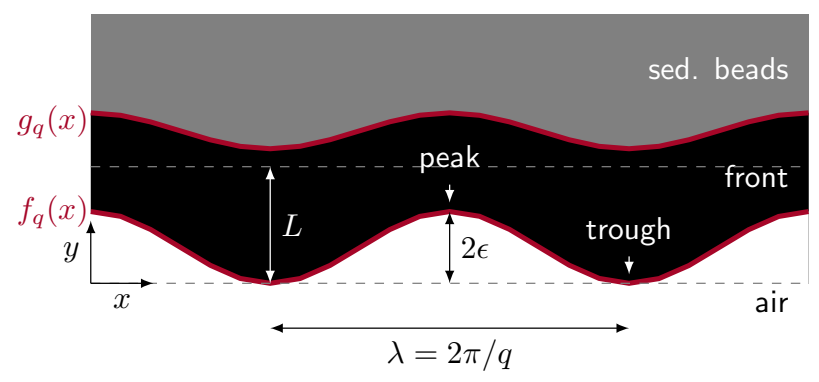

FIG. 7. (Color online) Illustration of the perturbation. The front is originally enclosed between the dashed lines. After the perturbation, it is enclosed between $g_{q}(x)$ and $f_{q}(x)$. A perturbation is considered unstable if the yield threshold at the peaks of the perturbations is lower than the threshold at the troughs, and otherwise stable. 
at the troughs of the perturbation, i.e., at $\cos (q x)=-1$. Otherwise, the interface at the troughs will move before the peaks, and the perturbation will flatten out.

Let $p_{p}$ and $p_{t}$ be the pressure threshold of Eq. (16) (evaluated at equality), for the peaks and the troughs respectively. We introduce the stability criterion function

$$
\Gamma(q)=\frac{p_{p}-p_{t}}{C},
$$

where $C$ is a positive constant independent of $q$, which will be determined later. $\Gamma$ is analogous to the negative of the growth rate of the perturbation, as used in linear stability analysis on systems where the dynamics are defined. The condition for the growth of the perturbation is now given by $\Gamma(q)<0$, and the most unstable perturbation wave vector $q^{*}$ is given by the minimum of $\Gamma(q)$, such that $\Gamma\left(q^{*}\right) \leqslant \Gamma(q)$.

We assume that the infinitesimal displacement results in infinitesimal pressure threshold variations, $p_{p}=p_{0}+\delta p_{p}$ and $p_{t}=p_{0}+\delta p_{t}$, where $\delta p_{p}$ and $\delta p_{t}$ are the changes of the threshold pressure induced by the perturbation at the peak and trough respectively, and $p_{0}$ is the threshold of the initial flat interface. We can therefore rewrite Eq. (18) as

$$
\Gamma(q)=\frac{\delta p_{p}-\delta p_{t}}{C} .
$$

The pressure variations can be written in terms of changes in the curvature $\delta \kappa$ and in the front length $\delta L$. We can expand Eq. (16), again evaluated at equality, to first order in $\delta \kappa$, and $\delta L$,

$$
\begin{aligned}
\delta p= & \frac{\partial p}{\partial \kappa} \delta \kappa+\frac{\partial p}{\partial L} \delta L \\
= & \left(\gamma+\sigma_{T} e^{L / \xi} L \frac{1-K_{2}}{(1+\kappa L)^{K_{2}}}\right) \delta \kappa \\
& +\sigma_{T} e^{L / \xi}(1+\kappa L)^{1-K_{2}}\left(\frac{1}{\xi}+\kappa \frac{1-K_{2}}{1+\kappa L}\right) \delta L .
\end{aligned}
$$

Note that $\sigma_{T}$, which is the stress threshold at the separation between the front and the sedimented region, is assumed to be constant and independent of $L$ and $\kappa$. Using this we have that Eq. (19) can be written as

$$
\begin{aligned}
\Gamma(q)= & \frac{1}{C}\left(\gamma+\sigma_{T} e^{L / \xi} L \frac{1-K_{2}}{(1+\kappa L)^{K_{2}}}\right)\left(\delta \kappa_{p}-\delta \kappa_{t}\right) \\
& +\sigma_{T} e^{L / \xi}(1+\kappa L)^{1-K_{2}}\left(\frac{1}{\xi}+\kappa \frac{1-K_{2}}{1+\kappa L}\right) \\
& \times\left(\delta L_{p}-\delta L_{t}\right),
\end{aligned}
$$

where $\delta L_{p}$ and $\delta L_{t}$ are the changes in the front length at the peak and the trough respectively, and similarly for the changes in the curvature $\delta \kappa_{p}$ and $\delta \kappa_{t}$.

The curvature of the perturbation is given by the negative of the second derivative of $f_{q}(x)$, to first order in $\epsilon$. The curvature is 0 for the straight segment, and after the perturbation,

$$
\delta \kappa=-f_{q}^{\prime \prime}(x)+O\left(\epsilon^{2}\right)=\epsilon q^{2} \cos (q x)+O\left(\epsilon^{2}\right) .
$$

The difference between the changes of the curvature at the peak $\delta \kappa_{p}$ and the changes at the trough $\delta \kappa_{t}$ is therefore

$$
\delta \kappa_{p}-\delta \kappa_{t}=2 \epsilon q^{2} .
$$

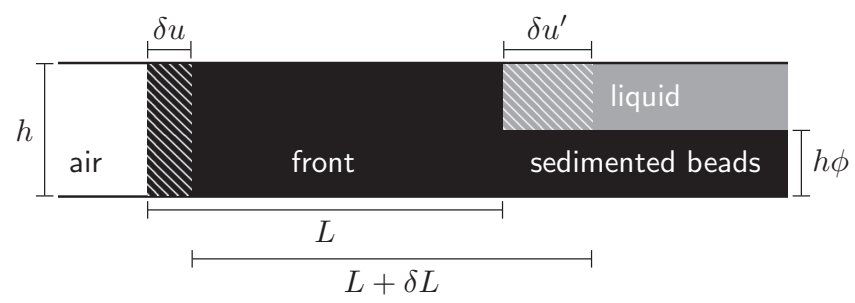

FIG. 8. Schematic representation of the cross section of the cell. The front is assumed to be incompressible. A volume associated to a displacement of a straight air-front interface $(\kappa=0)$, shown by the white striped pattern, is therefore coupled to an equal volume associated to the displacement of the front-liquid boundary (the outer boundary in Fig. 7). The regions which are marked as "liquid" and "sedimented beads" corresponds to the "sed. beads" in Fig. 7. The accumulation of the sedimented beads results in the increased displacement of the front-suspension boundary; $\delta u^{\prime}=\delta u /(1-\phi)$ and $\delta L=\delta u \phi /(1-\phi)$.

We now need to express the difference of front length changes, between the peak and the trough, $\delta L_{p}-\delta L_{t}$. Let $g_{q}(x)$ be the path which separates the front from the sedimented layer of beads, as shown in Fig. 7, such that the front length after the perturbation is given by $g_{q}(x)-f_{q}(x)$. The perturbation gives rise to a displacement field of the front, $\vec{d}(x, y)$; we will use this displacement field to find $g_{q}(x)$. We assume for simplicity that the displacement field is irrotational, $\nabla \times \vec{d}=0$, and incompressible, $\nabla \cdot \vec{d}=0$. We can therefore write the displacement as the negative of the gradient of a harmonic field $\psi_{q}(x, y)$, i.e., we have $\nabla^{2} \psi_{q}=0$, and $\vec{d}=-\nabla \psi_{q}$. We are only interested in displacements in the first order of $\epsilon$, and we can limit ourselves to the $y$ component of the deformation.

One can verify that the following field is harmonic:

$$
\psi_{q}(x, y)=-\epsilon y+\frac{\epsilon}{q} e^{-q y} \cos x q .
$$

Note also that the $y$ component of the displacement corresponds to the perturbation when evaluated at $y=0$,

$$
-\left.\frac{\partial}{\partial y} \psi_{q}\right|_{y=0}=f_{q}(x) .
$$

The separation path $g_{q}(x)$ between the front and the sedimented region, is given by the displacement field $\vec{d}(x, y)$ at $y=L$. As the separation path moves outwards it also accumulates new beads to the front. We can simply increase the displacement of the separation path between the front and the sedimented beads by a factor $1 /(1-\phi)$ to account for the bead accumulation, as shown in Fig. 8. The separation path $g_{q}(x)$, given the perturbation at the air-front interface, is therefore

$$
\begin{aligned}
g_{q}(y) & =L-\left.\frac{1}{1-\phi} \frac{\partial \psi_{q}}{\partial y}\right|_{y=L}+O\left(\epsilon^{2}\right) \\
& =L+\frac{\epsilon}{1-\phi}\left[1+e^{-L q} \cos (q x)\right]+O\left(\epsilon^{2}\right) .
\end{aligned}
$$




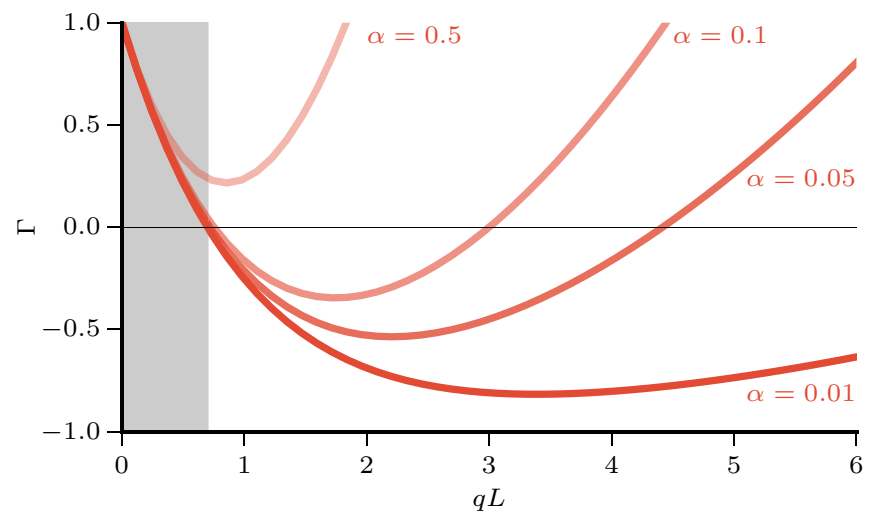

FIG. 9. (Color online) Stability criterion function $\Gamma$ [Eq. (29)] versus the product of the wave number and the front length $q L$ for various values of $\alpha$. The normalized filling fraction is set to $\phi=0.5$. The gray shaded region corresponds to $q L<-\ln (1-\phi)$, which is a stable region, independent of $\alpha$ [see Eq. (31)].

The change in front length along the perturbation is given by

$$
\begin{aligned}
\delta L & =g_{q}(x)-f_{q}(x)-L+O\left(\epsilon^{2}\right) \\
& =\epsilon\left[\frac{\phi}{1-\phi}+\left(\frac{e^{-L q}}{1-\phi}-1\right) \cos (q x)\right]+O\left(\epsilon^{2}\right) .
\end{aligned}
$$

The difference between the change of the front length at the peak $\delta L_{p}$ and the changes at the trough $\delta L_{t}$ is

$$
\delta L_{p}-\delta L_{t}=2 \epsilon\left(\frac{e^{-L q}}{1-\phi}-1\right) .
$$

We can now rewrite Eq. (21). We choose $C=2 \epsilon \sigma_{T} e^{L / \xi} / \xi$, to make $\Gamma(q)$ dimensionless. By using Eqs. (23) and (28), we get

$$
\Gamma(q)=\alpha(L q)^{2}+\frac{e^{-L q}}{1-\phi}-1
$$

where,

$$
\alpha=\frac{\xi}{L}\left(\frac{\gamma / L}{\sigma_{T} e^{L / \xi}}+1-K_{2}\right) .
$$

The first term in the parentheses in Eq. (30), is of order $\sim 10^{-2}$ when we use the approximate value of $L=3 \mathrm{~mm}$ (see Fig. 2), and the values in Table I. This means that high values of $q L$ are unconditionally unstable for $K_{2}>1+10^{-2} \simeq 1$, as $\alpha$ is negative. We will assume that $K_{2}<1$ for straight front segments, and therefore $\alpha>0$, in the subsequent discussion. Plots of $\Gamma$ versus $q L$, for different values of $\alpha$, and $\phi=0.5$, are shown in Fig. 9 . Note that $\Gamma(q=0)=\phi /(1-\phi)$, and that variations of $\phi$ change the behavior in the range of low $q L$. Variations of $\phi$ are unimportant for larger $q L$, as $e^{-q L} \rightarrow 0$. Note also that the stability criterion function is always stable for low wave numbers; $\Gamma$ is positive when

$$
\frac{e^{-L q}}{1-\phi}>1 \Rightarrow-\ln (1-\phi)>q L .
$$

This stable region is identified as the gray shaded region in Fig. 9. The stability in the low range of $q L$ is imposed by the filling fraction $\phi$, whereas the stability for high $q L$ is imposed by the effective surface tension $\gamma$, through $\alpha$ [Eq. (30)].

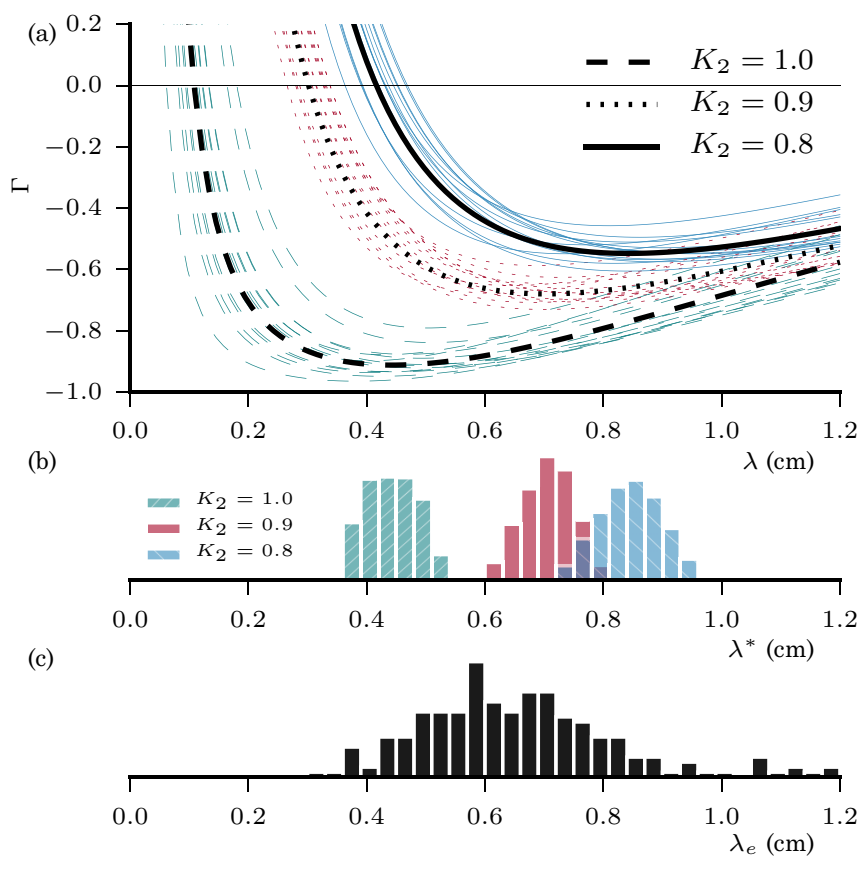

FIG. 10. (Color online) (a) Stability criterion function $\Gamma$, as defined in Eq. (29), vs the wavelength $\lambda=2 \pi / q$. A wavelength is unstable if $\Gamma(\lambda)<0 . \Gamma(\lambda)$ is drawn 15 times, to visualize the sensitivity to the parameters $\sigma_{T}, \xi, \gamma$, and $L$. For each realization, the parameters are drawn from uncorrelated uniform distributions on the interval defined by $\pm 15 \%$ of the mean value, $L=0.3 \mathrm{~cm}$, $\gamma=60 \mathrm{mN} / \mathrm{m}, \xi=0.06 \mathrm{~cm}$, and $\sigma_{T}=10 \mathrm{~Pa}$ in accordance with Table I. The filling fraction is fixed at $\phi=0.5$, and $K_{2}=1.0,0.9$, and 0.8 , for the green dashed, red dotted, and blue solid lines respectively. The thick black dashed/dotted/solid lines correspond to the mean values of the parameters, for each value of $K_{2}$. (b) Histograms of the theoretically estimated wavelengths $\lambda^{*}$, which minimize $\Gamma$ (i.e., the most unstable wavelength), based on $10^{5}$ realizations similar to the one plotted in (a), and for the three values of $K_{2}$. (c) Experimental observations of the wavelength $\lambda_{e}$ of the undulations. Estimated by measuring the linear peak to peak distance in the experimental pictures (see arrows in Fig. 2). This histogram is based on 214 measurements.

The wave number $q^{*}$, which minimizes $\Gamma$, can be written in terms of Lambert's $W$ function [36], which is implicitly defined by $y=W(y) e^{W(y)}$. We have that

$$
\begin{aligned}
\Gamma^{\prime}\left(q^{*}\right) & =2 \alpha L^{2} q^{*}-L \frac{e^{-L q^{*}}}{1-\phi}=0 \\
\Rightarrow q^{*} & =\frac{1}{L} W\left[\frac{1}{2 \alpha(1-\phi)}\right] .
\end{aligned}
$$

This wave number corresponds to the most unstable wavelength, defined by $\lambda^{*}=2 \pi / q^{*}$. Lambert's $W(y)$ function is monotonically increasing for growing positive arguments [36]. As the argument of $W$ in Eq. (32) is increasing with increasing $\phi$, higher $\phi$ generally corresponds to a smaller wavelength $\lambda^{*}$.

Different realizations of $\Gamma$ [Eq. (29)] versus the wavelength $\lambda=2 \pi / q$ are plotted in Fig. 10(a), for different values of $K_{2}$. The plot illustrates also how $\Gamma$ is sensitive to variations in the parameters, by superposing realizations with varying parameters $\xi, L, \sigma_{T}$, and $\gamma$. A histogram of the corresponding 
most unstable wavelengths $\lambda^{*}$ is shown in Fig. 10(b). These results are compared to a histogram of experimental estimates of the wavelengths of the undulations along the bubble interfaces $\lambda_{e}$ in Fig. 10(c). The experimental estimate is based on the measured linear peak to peak distance; examples of these peaks are shown by the arrows in Fig. 2.

\section{DISCUSSION}

The linear stability analysis above has omitted a number of complicating factors. We have for example left out the effect of the initial curvature of the interface, by considering a straight segment. We have also ignored the complications of the intermittency, and the locality of the deformation, by assuming harmonic perturbations. Moreover, we have assumed that the parameters in Table I, the front length $L$, and $K_{2}$ all are constant along the interface, although they may very well be subject to systematic variations. For these reasons, the linear stability analysis is only expected to give a first order approximation. In light of the expected accuracy of the prediction, we conclude that the prediction of the linear analysis agrees well with the experimental results, for $0.8<K_{2}<1.0$ (Fig. 10). We note that the most unstable wavelength increases with a decreasing $K_{2}$. An additional averaging over the range of $K_{2}$ will make the histograms of the theoretically estimated $\lambda^{*}$ [Fig. 10(a)], closer to the histogram of the experimentally observed $\lambda_{e}$ [Fig. 10(c)].

The range of plausible $K_{2}$ parameters, in agreement with the linear stability analysis (Fig. 10), is consistent with numerical estimates from discrete element method simulations [35], which estimated $K_{2}=0.8 \pm 0.1$ for a straight moving interface with a similar geometry. The values of $K_{2}$ may, however, change with the curvature of the front. This is analogous to variation of the Janssen parameter in silo experiments; the Janssen parameter is highly sensitive to the packing procedure [30]. The packing geometry of the beads in the front in our experiments may be a result of the curvature of the interface as it moves into the cell.

We conjecture that $K_{2}$ increase with decreasing curvatures, i.e., fronts adjacent to a convex interface [see Fig. 4(a)] develop a $K_{2}$ which is smaller than fronts adjacent to concave interface [see Fig. 4(b)]. This qualitative relation is suggested by the following two-dimensional simulation of the compaction of initially uniformly displaced discs inside an annulus shown in Fig. 11. The beads are either slowly compacted by the outward motion of the inner boundary [Fig. 11(a)], or by the inward motion of the outer boundary [Fig. 11(b)]. The simulation is made using the soft sphere discrete element method code MercuryDPM [37], assuming a damped linear spring interaction between particles in the normal direction, and damped linear spring sliders in the tangential direction. The walls are modelled as rough, i.e., particles cannot rotate while in contact with the boundary. Shaded connections in Fig. 11 indicate contacts with more than double the average contact force. The figure suggests that the force chains, indicated by consecutive black connections, tend to align with the radial direction when the inner boundary moves outwards, and orthoradially (tangentially to the circle), if the beads are compacted by the inward motion of the outer boundary. This suggests that the average effective normal stress is higher in the tangential direction (compared to the radial stress) when the

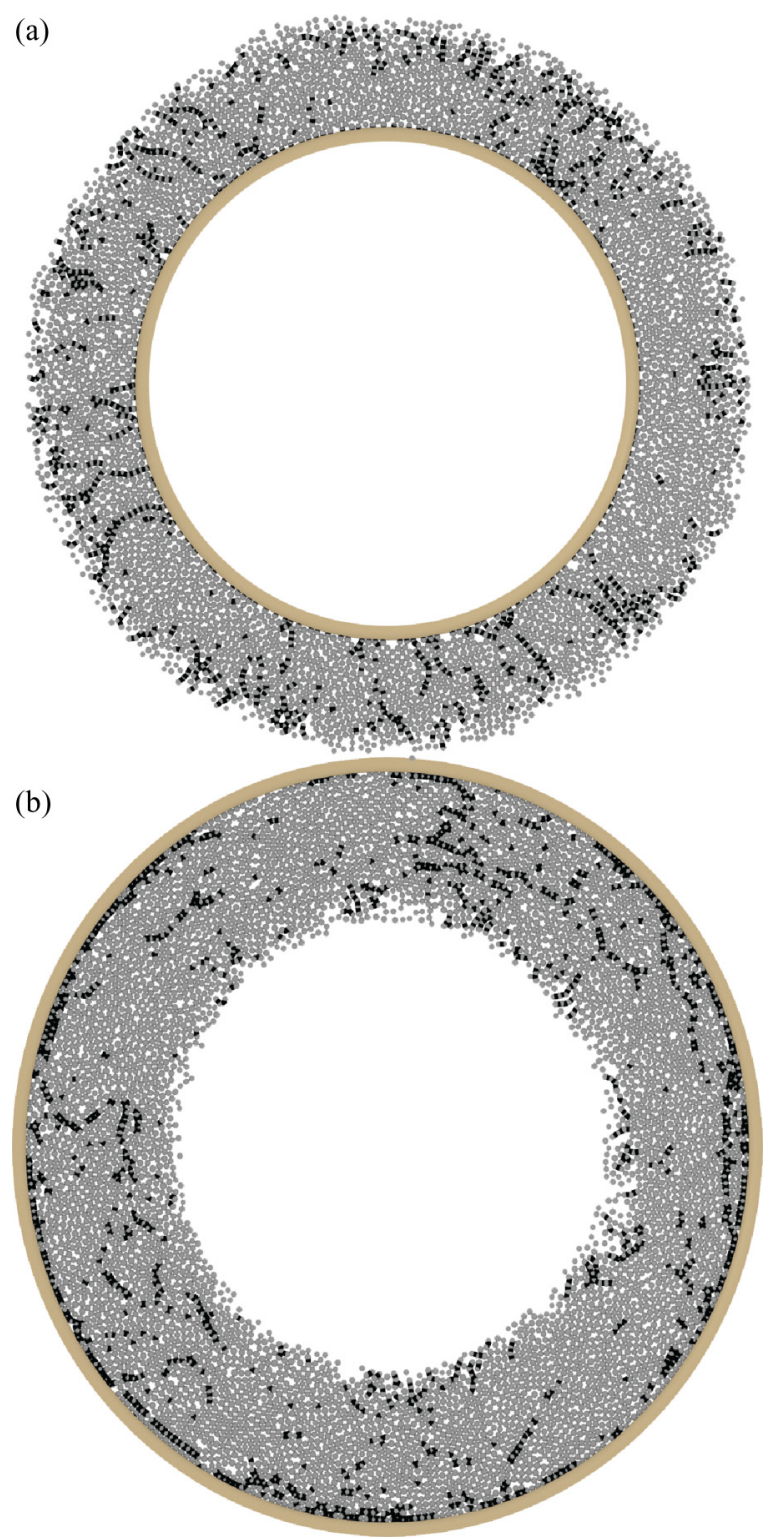

FIG. 11. (Color online) Compaction of beads in two dimensions. Black connections indicate contacts with more than double the average contact force. Contacts are overlaid for 50 consecutive time steps of the simulation. The beads are compacted as (a) the inner boundary moves outwards or as (b) the outer boundary slowly moves inwards. Note how the chains of contacts tend to orient radially in (a), which suggests that the average stress in the radial direction $\sigma_{r r}$ is bigger than the stress in the orthoradial direction $\sigma_{\theta \theta}$, i.e., $K_{2}<1$. In contrast, the chains tend to orient orthoradially in (b), which suggests that $\sigma_{\theta \theta}>\sigma_{r r}$ and $K_{2}>1$.

outer boundary moves inwards $\left(K_{2}>1\right)$, and that the radial average effective stress is higher when the inner boundary move outwards $\left(K_{2}<1\right)$, if we assume that the bulk part of the stress is mediated by force chains. The simulations are not meant to be a faithful representation of the compaction of the front, as the front is three dimensional. In addition, gravity is likely to affect how the beads in our experiments settle down, so we cannot use estimated values from the two-dimensional simulations directly. We assume, however, that the general 


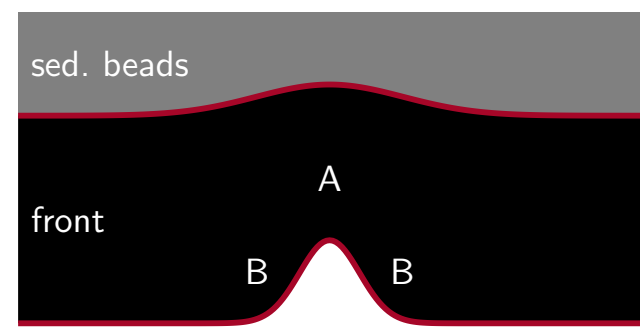

air

FIG. 12. (Color online) Schematic of the channeling of air through the front. Consider two regions of the front. A: The front adjacent to the tip of the channel is compacted as an interface segment of high curvature $\kappa$ moves outwards (towards the sedimented beads). $\mathrm{B}$ : The front at the shoulders of the channel are compacted as the negatively curved interface moves outwards. Undulations along the interface are not shown in the figure.

direction of the force chains in the front, in the $(r, \theta)$ plane, is similar to that of the two-dimensional simulations (Fig. 11). We will therefore assume that $K_{2}$ is a decreasing function of $\kappa$.

This curvature dependence of $K_{2}$ may be of importance when the yield threshold [Eq. (16)] is applied to the interface of the channels which make their way through the front of a bubble, shown in Fig. 12. The channel configurations contain regions of high positive curvatures, region marked A in Fig. 12, and with low negative curvatures, region marked B in Fig. 12. If we assume that $K_{2}$ grows beyond 1 in the B region, and that $K_{2}$ takes a value below 1 in the A region, the yield stress of the front will behave qualitatively differently in the different regions, as shown in Fig. 6. The growing yield threshold for decreasing curvatures in region B [Fig. 6(b)] may therefore result in arching effects, and prevent the front from further deformation. Note that the channel growth is well beyond the presented linear stability analysis. It is hard to determine the experimental values of $\kappa$ at interfaces which correspond to region $\mathrm{B}$; it is possible that these configurations are mobile, and correspond to $\kappa L<-1$ (see Fig. 4). In that case, we may need to modify the theoretical framework further. We leave the details of these mechanisms for future work.

In summary, we have derived a natural extension to the yield stress model of bead fronts, used to simulate frictional finger structures $[15,22]$. The new expression for the yield pressure threshold [Eq. (16)] incorporates the tangential stress component, and the geometrical modifications due to the curvature of the front, by assuming a linear relationship between the radial and the tangential stress, $\sigma_{v v}=K_{2} \sigma_{u u}$ (or $\sigma_{\theta \theta}=K_{2} \sigma_{r r}$ in cylindrical coordinates). These modifications are important for the bubble dynamics shown in Fig. 1, as $\kappa L$ takes values which cannot be neglected, in particular at the channels between the bubbles.

We have also presented a linear stability analysis for a straight front segment, based on the threshold criterion in Eq. (16). This linear stability analysis gives a closed form expression for the most unstable wave numbers in Eq. (32). The numerical values of the wavelengths agree with the wavelengths of the undulations seen along the interface of bubbles in experiments, for reasonable choices of parameters. In particular, the results are consistent with $0.8<K_{2}<1$ (Fig. 10).

The theoretical results we have presented will be of importance for future attempts to simulate the bubble formation. The discretization scheme presented in Ref. [38] provides a natural framework for such a simulation. Such simulations may also need to take into account the $K_{2}$ dependence of the curvature, to faithfully represent the channeling through the front around the bubbles (Fig. 12). We suggest that this dependence can be determined from a three-dimensional bead simulation based on the discrete element method, similar to the two-dimensional example in Fig. 11.

\section{ACKNOWLEDGMENTS}

We thank E. Flekkøy and K. J. Måløy for fruitful discussions. J.A.E. acknowledges support from the Research Council of Norway (NFR) through the NFR Project No. 200041/S60, and from Campus France through the Eiffel Grant. B.M. acknowledges support from NFR Grant No. 213462/F20. B.S. acknowledges support from EPSRC Grant No. EP/L013177/1. R.T. acknowledges support from The European Union SEVENTH FRAMEWORK PROGRAMME for research, technological development, and demonstration under Grant Agreement No. 316889 FlowTrans. R.T. acknowledges additional support from the University of Oslo and Université de Strasbourg.
[1] G. Varas, J.-C. Géminard, and V. Vidal, Granular Matter 15, 801 (2013).

[2] I. B. Belien, K. V. Cashman, and A. W. Rempel, Earth Planet. Sci. Lett. 297, 133 (2010).

[3] L. Cueto-Felgueroso and R. Juanes, Phys. Rev. E 79, 036301 (2009).

[4] M. Sahimi, Rev. Mod. Phys. 65, 1393 (1993).

[5] B. Koppelman, A. Walker, and E. Woods, Shale gas extraction in the UK: A review of hydraulic fracturing 2012, The Royal Society, The Royal Academy of Engineering (unpublished).

[6] A. J. Cavanagh and R. S. Haszeldine, Int. J. Greenhouse Gas Control 21, 101 (2014).

[7] C. Chevalier, A. Lindner, and E. Clement, Phys. Rev. Lett. 99, 174501 (2007).
[8] O. Johnsen, R. Toussaint, K. J. Måløy, and E. G. Flekkøy, Phys. Rev. E 74, 011301 (2006).

[9] Ø. Johnsen, C. Chevalier, A. Lindner, R. Toussaint, E. Clément, K. Måløy, E. Flekkøy, and J. Schmittbuhl, Phys. Rev. E 78, 051302 (2008).

[10] X. Cheng, L. Xu, A. Patterson, H. M. Jaeger, and S. R. Nagel, Nat. Phys. 4, 234 (2008).

[11] X.-Z. Kong, W. Kinzelbach, and F. Stauffer, Chem. Eng. Sci. 65, 4652 (2010).

[12] A. Debacker, S. Makarchuk, D. Lootens, and P. Hébraud, Phys. Rev. Lett. 113, 028301 (2014).

[13] M. Niebling, R. Toussaint, E. G. Flekkøy, and K. J. Måløy, Phys. Rev. E 86, 061315 (2012). 
[14] R. Niebling, R. Toussaint, E. G. Flekkøy, and Måløy, Rev. Cub. Fis. 29, 1E66 (2012).

[15] B. Sandnes, E. G. Flekkøy, H. A. Knudsen, K. J. Måløy, and H. See, Nat. Commun. 2, 288 (2011).

[16] C. Chevalier, A. Lindner, M. Leroux, and E. Clement, J. NonNewtonian Fluid Mech. 158, 63 (2009).

[17] R. Holtzman, M. L. Szulczewski, and R. Juanes, Phys. Rev. Lett. 108, 264504 (2012)

[18] A. Islam, S. Chevalier, I. B. Salem, Y. Bernabe, R. Juanes, and M. Sassi, Int. J. Multiphase Flow 58, 279 (2014).

[19] H. Shin and J. C. Santamarina, Earth Planet. Sci. Lett. 299, 180 (2010).

[20] B. Sandnes, E. G. Flekkøy, K. J. Måløy, and J. A. Eriksen, Rev. Cub. Fis. 29, 1E23 (2012).

[21] B. Sandnes, H. A. Knudsen, K. J. Måløy, and E. G. Flekkøy, Phys. Rev. Lett. 99, 038001 (2007).

[22] H. A. Knudsen, B. Sandnes, E. G. Flekkøy, and K. J. Måløy, Phys. Rev. E 77, 021301 (2008).

[23] J. A. Eriksen, R. Toussaint, E. G. Flekkøy, K. J. Måløy, and B. Sandnes (unpublished).

[24] B. Sandnes, E. Flekkøy, and K. Måløy, Eur. Phys. J.: Spec. Top. 204, 19 (2012).

[25] See Supplemental Material at http://link.aps.org/supplemental/ 10.1103/PhysRevE.91.052204 for videos of the bubble behavior.
[26] H. Janssen, Z VER DTSCH ING 39, 1045 (1895).

[27] The Janssen parameter was denoted by $\kappa$ in Refs. [21,22]. We use here $\kappa$ for the curvature and $K_{1}$ for the Janssen parameter.

[28] R. M. Nedderman, Statics and Kinematics of Granular Materials (Cambridge University Press, Cambridge, England, 2005).

[29] P.-G. de Gennes, Rev. Mod. Phys. 71, S374 (1999).

[30] G. Ovarlez, C. Fond, and E. Clément, Phys. Rev. E 67, 060302 (2003).

[31] W. M. Lai, D. H. Rubin, D. Rubin, and E. Krempl, Introduction to Continuum Mechanics, Forth edition, (Butterworth-Heinemann, Burlington, MA, 2009).

[32] J.-P. Bouchaud, M. Cates, and P. Claudin, J. Phys. I 5, 639 (1995).

[33] J. Wittmer, M. Cates, and P. Claudin, J. Phys. I 7, 39 (1997).

[34] J. P. Wittmer, P. Claudin, M. E. Cates, and J.-P. Bouchaud, Nature (London) 382, 336 (1996).

[35] B. Marks, B. Sandnes, G. Dumazer, J. A. Eriksen, and K. J. Måløy, arXiv:1505.04054.

[36] R. M. Corless, G. H. Gonnet, D. E. Hare, D. J. Jeffrey, and D. E. Knuth, Adv. Comput. Math. 5, 329 (1996).

[37] A. Thornton, T. Weinhart, S. Luding, and O. Bokhove, Int. J. Mod. Phys. C 23, 1240014 (2012).

[38] J. A. Eriksen, R. Toussaint, E. G. Flekkøy, K. J. Måløy, and B. Sandnes (unpublished). 